\title{
Comparison of protein expression in two prostate cancer cell-lines, LNCaP and DU145, after treatment with somatostatin
}

\author{
ZHAOXU LIU ${ }^{1,2}$ MARCELA MARQUEZ ${ }^{1}$, STEN NILSSON $^{1}$ and ANDERS R. HOLMBERG ${ }^{1}$ \\ ${ }^{1}$ Department of Oncology and Pathology, Karolinska Institute, SE-171 76 Stockholm, Sweden; ${ }^{2}$ Department \\ of Urology, Qilu Hospital/School of Nursing, Shandong University, 250012 Jinan, P.R. China
}

Received April 14, 2009; Accepted July 1, 2009

DOI: $10.3892 /$ or_00000587

\begin{abstract}
The mechanisms underlying prostate cancer progression are poorly understood. Proteins responsive to androgens may be involved in the development and progression of prostate cancer and the ultimate failure of androgen-ablation therapy. Therapy with somatostatin (sms) analogues could be a possible therapeutic alternative to chemotherapy in hormone refractory prostate cancer patients. We used two prostate cancer cell-lines, LNCaP (androgendependent) and DU145 (androgen-independent), to compare the protein expressions. Both cell lines were treated with sms and its derivative smsdx. Smsdx is a glycosylated poly sms with high stability suitable for clinical use. A comparison study of protein expression was analyzed by means of twodimensional gel electrophoresis (2DE) followed by mass spectrometric analysis. Marked quantitative differences were observed in the protein expression profiles in sms/smsdx treated LNCaP and DU145 cells compared to the control cells. One third of the detected proteins were differentially expressed (PRDXs, hnRNPs, HSPs, RKIP). Concordance in protein expression patterns was observed between smsdx and sms treated cells with strong agreement between the up- and down-regulation of proteins. Fifty-eight (isoforms of 49 proteins) protein spots were identified and found differentially expressed at 2-fold change between LNCaP and DU145 cells. Thirty-one proteins in $\mathrm{LNCaP}$ have higher expressions than in DU145. Twenty-seven proteins in DU145 have higher expressions than in LNCaP. Most of the differentially expressed proteins (2-fold) between LNCaP and DU145 cells were affected by sms/smsdx treatment (1.2- to 2.6fold change). Sms/smsdx affects the mitochondria of prostate cancer cells in a way that eventually triggers mitochondrial-
\end{abstract}

Correspondence to: Dr Zhaoxu Liu, Department of Urology, Qilu Hospital/School of Nursing, Shandong University, 250012 Jinan, P.R. China

E-mail: zhaoxu.liu@ki.se

Key words: 2-DE, mass spectrometry, prostate cancer, protein expression, somatostatin mediated apoptosis. Regulation of certain proteins (e.g., RKIP, VDACs) by sms/smsdx suggests that sms/smsdx exerts its effects on prostate cancer cells via MAPK pathway and by regulating the activities of phosphotyrosine phosphatases.

\section{Introduction}

Prostate cancer is the most common malignancy and the second leading cause of cancer-related mortality in men in the Western world (1). Although androgen ablation is the most effective therapy for patients with advanced prostate cancer, progression to androgen independence (AIPC) and hormone refractory (HRPC) eventually occurs (2). Androgenindependent prostate cancer carries a bleak prognosis with short survival time. AIPC metastasizes preferentially to the skeleton which is associated with considerable morbidity such as pain and fractures.

Several pathways provide insights into the mechanism of androgen action and schemes by which cancer cells subvert normal growth control and escape treatment attempts. Understanding the pathways that lead to AIPC will eventually lead to the development of new therapies. In our previous studies $(3,4)$, smsdx (glycosylated poly sms with high stability suitable for clinical use) was found to be able to trigger upregulation of catalytic mitochondrial proteins and seemed to affect apoptotic-related proteins in androgen-dependent (AD) prostate cancer LNCaP cells. One hypothesis could be that there are groups of proteins crucially involved in the progression and transition to HRPC. Given the importance of proteins regulated by sms/smsdx in the disease development and progression, characterization of the affected proteins may provide candidates for biomarkers and elucidate the regulatory mechanisms of sms/smsdx effects.

The aim of this study was to compare the protein expression in androgen-dependent and androgen-independent prostate cancer cells after sms and smsdx treatment. Through proteomic analysis, we could explore potential candidate biomarkers and elucidate sms/smsdx effect pathways in prostate cancer.

\section{Materials and methods}

Cell culture. LNCaP and DU145 human prostate cancer cell lines (American Type Culture Collection, Rockville, MD, 
Table I. The RT-PCR primers sequences and amplified genes.

\begin{tabular}{llll}
\hline Amplified gene & Primers & Sequence & Product (bp) \\
\hline PRDX2 & Sense & 5'-GCCCACGCAGCTTTCAGTCA-3' & 623 \\
& Antisense & 5'-AGCCAGCCTAATTGTGTTTG-3' & \\
TCTP & Sense & 5'-GAGGGGAAGATGGTCAGTAGG-3' & 278 \\
& Antisense & 5'-TGCTTGATTTGTTCTGCAGC-3' & 576 \\
HSP27 & Sense & 5'-CCAGAGCAGAGTCAGCCAGCAT-3' & \\
\hline
\end{tabular}

USA) were cultured in RPMI-1640 containing $10 \%$ foetal bovine serum (FBS), $2 \mathrm{mM}$ glutamine and $100 \mathrm{IU} / \mathrm{ml}$ penicillin-100 $\mu \mathrm{g} / \mathrm{ml}$ streptomycin at $37^{\circ} \mathrm{C}$ in a humidified atmosphere of $5 \% \mathrm{CO}_{2}$. The medium was changed two times a week and the cells were trypsinized and subcultivated once a week. Smsdx was prepared as described previously (5). Sms was from Ferring, Kiel, Germany. The cell culture was treated with smsdx or with sms for three days, $1 \mathrm{nM}$ per day, as described by Brevini et al (6). Controls were untreated cells (negative) and cells treated with sms14 (positive).

Sample preparation and protein extraction and concentration. All the cultured cells were prepared according to a cell preparation method (7), with slight modification. Briefly, the cell monolayers were washed twice in phosphate-buffered saline (PBS) and then scraped off in ice-cold PBS including protease inhibitors (PIH), phenylmethylsulfonyl fluoride (PMSF) $0.2 \mathrm{mM}$ and $0.83 \mathrm{mM}$ benzamidine pelleted at $660 \mathrm{x} \mathrm{g}$, $3 \mathrm{~min}\left(4^{\circ} \mathrm{C}\right)$ and washed one time before final centrifugation at $2700 \mathrm{x} \mathrm{g}, 5 \mathrm{~min}$. The wet weight of the cell pellet was recorded and the cells were stored at $-80^{\circ} \mathrm{C}$ until further processing. Each sample was then dissolved lysis buffer containing $8 \mathrm{M}$ urea, $4 \%$ SDS, reducing agents and protease inhibitors. Protein determination was made using Pierce BCA protein assay reagent (Rockford, IL, USA).

Electrophoresis, scanning and image analysis. 2-DE was performed as previously described (7). Pharmalyte 3-10 for IPG strip 3-10 were used for isoelectric focusing, and 12.5\% linear gradient SDS-polyacrylamide gels were used in the second dimension. Gels were stained with silver nitrate as described by Rabilloud et al (8) and scanned at $100-\mu \mathrm{m}$ resolution using a Molecular Dynamics (Sunnyvale, CA, USA) laser densitometer. Both quantitative and qualitative 2-DE data sets were analyzed using PDQuest software, a 2-DE software analysis program (9) obtained from Pharmacia Biotech (Uppsala, Sweden).

Mass spectrometry analysis. Protein spots of $\mathrm{LNCaP}$ cells were selected for identification by mass spectrometry as described before (7). In short, proteins were identified with a vMALDILTQ instrument (Thermo Electron, San José, CA, USA). The spot picking, destaining, digestion, extraction, sample preparation and spotting on MALDI target plates were carried out using a spothandling workstation (ETTAN Spothandling workstation, GE Healthcare) and a standard protocol provided by GE Healthcare. The MS spectra were collected in the 9002000 Da mass range while the mass range for the MS/MS spectra were automatically selected by the system based on a Q-value of 0.25.

Protein identification. Database searches were done using both the MASCOT and Sequest search algorithm against the human session of the IPI protein database (version 2.38). The two searches were compared in the in-house developed software Promiscuous MS/MS. A minimum of two peptides and a Mascot score of 45 were required for a protein to be accepted as identified.

RNA extraction and RT-PCR. Total LNCaP cellular RNA, after treatment with sms and smsdx in different concentration $(0,0.1,1.0,10,50,100 \mathrm{nM}$ for $24 \mathrm{~h})$ and incubation time $(0,1,4,8,16,24 \mathrm{~h}$ for $10 \mathrm{nM})$, was extracted using the TRIzol $^{\circledR}$ (Invitrogen, Carlsbad, CA, USA), according to the manufacturer's instruction. cDNA was synthesized using random primers (N6) and MMLV reverse transcriptase. The PCR for PRDX2, TCTP, HSP27 mRNA were performed by using the following primer pairs (Table I).

A total of $32 \mathrm{PCR}$ cycles at $94^{\circ} \mathrm{C}$ for $30 \mathrm{sec}, 53^{\circ} \mathrm{C}$ for $40 \mathrm{sec}$ and $72^{\circ} \mathrm{C}$ for $60 \mathrm{sec}$ for PRDX2; at $94^{\circ} \mathrm{C}$ for $60 \mathrm{sec}, 60^{\circ} \mathrm{C}$ for $60 \mathrm{sec}$ for TCTP; at $94^{\circ} \mathrm{C}$ for $30 \mathrm{sec}, 57^{\circ} \mathrm{C}$ for $30 \mathrm{sec}$, and $72^{\circ} \mathrm{C}$ for $40 \mathrm{sec}$ for $\mathrm{HSP} 27$, and $72^{\circ} \mathrm{C}$ for $60 \mathrm{sec}$ for was carried out. B-actin expression was used as a control for RNA loading and reverse transcription efficiency and amplified with its specific primers using 25 cycles. PCR products were resolved in $2 \%$ agarose gels, stained with ethidium bromide, and visualized in UV light.

\section{Results}

Creation of $2 D E$ protein map of LNCaP. We successfully identified a set of 222 proteins (isoforms and variants) for 2DE map in LNCaP cell line. The highest and lowest protein molecular masses that we found were $111 \mathrm{kDa}$ for $150 \mathrm{kDa}$ oxygen-regulated protein (HYOU1) and 14.8 kDa for gastricassociated differentially-expressed protein YA61P (YA61). Proteins pI range from 4.59 to 10.52 .

Variation in protein expression between LNCaP and DU145 cell line. After analysis using PDQuest software version 7.0, 
Table II. Thirty-one lower expressed proteins (>2-fold) in DU145 than in LNCaP.

\begin{tabular}{|c|c|c|c|c|c|c|}
\hline Protein name & Short name & Uniprot ID & $\begin{array}{l}\text { DU145 } \\
\text { smsdx }\end{array}$ & $\begin{array}{l}\text { DU145 } \\
\text { sms }\end{array}$ & $\begin{array}{l}\text { LNCaP } \\
\text { smsdx }\end{array}$ & $\begin{array}{l}\mathrm{LNCaP} \\
\text { sms }\end{array}$ \\
\hline Creatine kinase, $\mathrm{B}$ chain & CKB & P12277 & 2.0 & 1.2 & -1.3 & - \\
\hline Septin 2 & S EPT2 & Q15019 & 2.0 & 2.0 & - & 1.6 \\
\hline $\begin{array}{l}\text { Serine-threonine kinase receptor- } \\
\text { associated protein }\end{array}$ & STRAP & Q9Y3F4 & -1.2 & -2.0 & 1.5 & - \\
\hline $\begin{array}{l}\text { Ubiquinol-cytochrome c reductase iron- } \\
\text { sulfur subunit }\end{array}$ & UQCRFS1 & P47985 & -1.5 & -1.2 & NA & - \\
\hline $\begin{array}{l}\text { Heterogeneous nuclear ribonucleoproteins } \\
\mathrm{C} 1 / \mathrm{C} 2\end{array}$ & HNRPC & P07910 & 1.5 & -1.5 & - & 2.6 \\
\hline Leucine aminopeptidase & LAP3 & Q6P0L6 & 1.2 & -1.2 & 1.5 & - \\
\hline Malate dehydrogenase & MDH2 & P40926 & 1.2 & -1.2 & - & 2.1 \\
\hline Voltage-dependent anion channel 1 & VDAC1 & P21796 & 1.5 & -1.5 & 1.7 & 1.7 \\
\hline Enabled protein homolog & ENAH & Q8N8S7 & -2.0 & 1.5 & 1.8 & 1.4 \\
\hline $78 \mathrm{kDa}$ glucose-regulated protein & GRP78 & P11021 & 1.2 & - & 1.3 & 1.3 \\
\hline Endoplasmin & HSP90B1 & P14625 & 1.5 & - & 2.3 & - \\
\hline Far upstream element binding protein 1 & FUBP1 & Q96AE4 & 1.2 & - & 1.3 & 1.3 \\
\hline Fibroblast growth factor-5 & FGF5 & P12034 & 1.2 & - & - & 2.8 \\
\hline Glycerol-3-phosphate dehydrogenase & GPD2 & P43304 & 1.2 & - & 1.6 & 1.7 \\
\hline Creatine kinase, ubiquitous & CKMT & P12532 & -2.0 & - & 1.4 & - \\
\hline Heat-shock protein $\beta-1$ & HSPB1 & P04792 & -1.2 & - & - & 1.3 \\
\hline Mitochondrial inner membrane protein & IMMT & Q16891 & -1.2 & - & - & 1.4 \\
\hline Protein disulfide-isomerase A3 & PDIA3 & P30101 & -1.2 & - & 1.2 & - \\
\hline ATP synthase $\gamma$ chain & ATP5C1 & P36542 & - & 1.2 & - & 3.3 \\
\hline Far upstream element binding protein 2 & KHSRP & Q92945 & - & 2.0 & - & - \\
\hline Flavin reductase & BLVRB & P30043 & - & 1.5 & -1.4 & - \\
\hline Heat-shock protein $B-1$ & HSPB1 & P04792 & - & 1.2 & 1.7 & -1.3 \\
\hline Heterogeneous nuclear ribonucleoprotein D0 & HNRPD & Q14103 & - & 1.5 & - & 1.8 \\
\hline Electron transfer flavoprotein $\alpha$-subunit & ETFA & P13804 & - & -2.0 & - & 1.4 \\
\hline $\begin{array}{l}\text { Heterogeneous nuclear ribonucleoproteins } \\
\mathrm{C} 1 / \mathrm{C} 2\end{array}$ & HNRPC & P07910 & - & - & 2.9 & - \\
\hline Lupus La protein & SSB & P05455 & - & - & -1.6 & -1.3 \\
\hline $\begin{array}{l}\text { Probable mitochondrial import receptor } \\
\text { subunit TOM40 homolog }\end{array}$ & TOMM40 & O96008 & - & - & 2.6 & 2.1 \\
\hline Prostatic binding protein & PEBP1 & P30086 & - & - & -1.3 & - \\
\hline Acetyl-CoA acetyltransferase, cytosolic & ACAT2 & Q9BWD1 & - & - & - & NA \\
\hline Septin 11 & S EPT11 & Q9NVA2 & - & - & - & 1.2 \\
\hline Voltage-dependent anion channel 1 & VDAC1 & P21796 & - & - & - & 1.7 \\
\hline
\end{tabular}

Some of listed differentially expressed proteins in DU145 and LNCaP can be regulated by sms and/or smsdx in up- and down-regulated manner.

we found 344 protein spots were differentially expressed at 2-fold change between LNCaP and DU145 cells. One hundred and twenty-five protein spots in DU145 cells were matched with the reference protein map of LNCaP cells. Fifty-eight protein spots were identified and found differentially expressed at 2-fold change between LNCaP and DU145 cells. These spots represented different isoforms of 49 proteins. In general, the distribution of isoforms obtained in the present study was as follows: HnRPH1 was represented by three isoforms,
HSPB 1 by three, hnRPC by two, KRT8 by two, PSIP1 by two, TAF15 by two, VDAC1 by two.

Differential protein expression between LNCaP and DU145 cell line. Fifty-eight different protein expressions (2-fold change) were found between LNCaP and DU145 cells. Thirty-one proteins in LNCaP have higher expressions than in DU145. Twenty-seven proteins in DU145 have higher expressions than in LNCaP (Tables II and III). For example, 
Table III. Twenty-seven higher expressed proteins (>2-fold) in DU145 than in LNCaP.

\begin{tabular}{|c|c|c|c|c|c|c|}
\hline Protein name & Short name & Uniprot ID & $\begin{array}{l}\text { DU145 } \\
\text { smsdx }\end{array}$ & $\begin{array}{l}\text { DU145 } \\
\text { sms }\end{array}$ & $\begin{array}{l}\text { LNCaP } \\
\text { smsdx }\end{array}$ & $\begin{array}{l}\text { LNCaP } \\
\text { sms }\end{array}$ \\
\hline$\alpha$ enolase & ENO1 & P06733 & 1.5 & 1.5 & - & 1.3 \\
\hline Heat shock cognate $71 \mathrm{kDa}$ protein & HSPA8 & P11142 & 1.2 & 2.0 & -1.3 & - \\
\hline Lens epithelium-derived growth factor p 75 & PSIP1 & Q8N4N4 & 2.0 & 1.5 & 2.1 & 3.4 \\
\hline Paraspeckle protein 1 & PSPC1 & Q8WXF1 & 1.2 & 1.2 & 1.4 & 1.3 \\
\hline Thioredoxin-dependent peroxide reductase & PRDX3 & P30048 & 1.2 & 1.2 & 1.3 & - \\
\hline Cofilin, non-muscle isoform & CFL1 & $\mathrm{P} 23528$ & -1.2 & -1.5 & - & - \\
\hline Lens epithelium-derived growth factor p75 & PSIP1 & Q8N4N4 & -1.5 & -1.2 & - & 3.0 \\
\hline Glyoxalase I & GLO1 & Q04760 & -1.5 & -1.2 & - & -1.4 \\
\hline Mitochondrial processing peptidase $\beta$ subunit & РMPCB & O75439 & -2.0 & -1.2 & 1.2 & - \\
\hline Heterogeneous nuclear ribonucleoprotein $\mathrm{H}$ & HNRPH1 & P31943 & 1.2 & -1.2 & 1.9 & - \\
\hline Lamin B1 & LMNB1 & P20700 & -1.2 & 1.2 & 1.6 & - \\
\hline Dihydrolipoyl dehydrogenase & DLD & P09622 & 1.2 & - & - & 1.5 \\
\hline Keratin, type II cytoskeletal 8 & KRT8 & P05787 & -1.2 & - & 4.0 & 2.1 \\
\hline Keratin, type II cytoskeletal 8 & KRT8 & P05787 & - & -2.0 & - & 3.8 \\
\hline Peptidyl-prolyl cis-trans isomerase A & PPIA & P62937 & - & -1.5 & 1.2 & - \\
\hline Peroxiredoxin 2 & PRDX2 & P32119 & - & -1.2 & - & -2.1 \\
\hline Polyribonucleotide nucleotidyltransferase 1 & PNPT1 & Q9BRU3 & - & -1.5 & 2.8 & 1.8 \\
\hline Heat-shock protein $\beta-1$ & HSPB 1 & P04792 & - & - & - & -1.5 \\
\hline Heterogeneous nuclear ribonucleoprotein $\mathrm{H}$ & HNRPH1 & P31943 & - & - & -1.4 & - \\
\hline Heterogeneous nuclear ribonucleoprotein $\mathrm{H}$ & HNRPH1 & P31943 & - & - & - & 1.2 \\
\hline Heterogeneous nuclear ribonucleoproteins A2/B1 & HNRPA2B1 & P22626 & - & - & - & 2.7 \\
\hline Splicing factor, arginine/serine-rich 1 & SFRS1 & Q07955 & - & - & - & -1.7 \\
\hline Stress-70 protein & HSPA9 & P38646 & - & - & 1.8 & - \\
\hline TATA-binding protein associated factor $2 \mathrm{~N}$ & TAF15 & Q92804 & - & - & 1.6 & 1.5 \\
\hline TATA-binding protein associated factor $2 \mathrm{~N}$ & TAF15 & Q92804 & - & - & - & 2.6 \\
\hline UBA/UBX $33.3 \mathrm{kDa}$ protein & SAKS1 & Q04323 & - & - & -2.1 & -1.3 \\
\hline $\begin{array}{l}\text { Voltage-dependent anion-selective channel } \\
\text { protein } 2\end{array}$ & VDAC2 & P45880 & - & - & 1.8 & 1.8 \\
\hline
\end{tabular}

Some of listed differentially expressed proteins in DU145 and LNCaP can be regulated by sms and/or smsdx in up- and down-regulated manner.

prostatic binding protein (PEBP1/RKIP) has a higher level expression in androgen-dependent LNCaP cells than in androgen-independent DU145 cells.

Sms/smsdx affected proteins. After treatment with sms and smsdx, we found strong agreement with the protein expression regulation in DU145 cells (Table IV). Most of the differentially expressed proteins (>2-fold) between LNCaP and DU145 cells were affected by sms/smsdx treatment (1.2- to 2.6-fold change) (Tables II and III). Fig. 1 shows down-regulation of protein expression of PRDX2 (A) and TCTP (B) (2-fold) after treatment with $1 \mathrm{nM} \mathrm{sms/smsdx} \mathrm{for}$ three days in the prostate cancer cell line LNCaP.

$R T-P C R$ results. Of the identified differentially expressed proteins between LNCaP and DU145 cells, PRXII, TCTP, HSP27 were tested on the mRNA expression in LNCaP after treatment with sms and smsdx in different concentration and incubation time. Fig. 2 shows up- and mRNA downexpression of PRDX2 (A) and TCTP (B), respectively, after incubation with smsdx in different concentrations and incubation time in LNCaP cell line. PRDX2 was found to be up regulated in a dose-dependent manner. TCTP mRNA was down-regulated in the current study. HSP27 mRNA was also down-regulated (data not shown).

\section{Discussion}

Prostate cancer continues to be the most common malignancy diagnosed in men in the western countries. In Europe, there were about 190,000 new cases in 2004 (10), 301, 500 incident cases, and 87,400 deaths in 2006 (11). The transition of prostate cancer from androgen-dependent (ADPC) to androgen-independent (AIPC) and hormone refractory 
Table IV. Twenty-two proteins regulated by sms/smsdx ( 2-fold) in DU145.

\begin{tabular}{|c|c|c|c|c|}
\hline Protein name & Short name & Uniprot ID & smsdx & sms \\
\hline $60 \mathrm{kDa}$ heat shock protein & HSPD1 & P10809 & 2.0 & 2.0 \\
\hline Creatine kinase, $\mathrm{B}$ chain & CKB & P12277 & 2.0 & 1.2 \\
\hline Lens epithelium-derived growth factor p75 & PSIP1 & Q8N4N4 & 2.0 & 1.5 \\
\hline Septin 2 & SEPTII & Q15019 & 2.0 & 2.0 \\
\hline$\delta 3,5$ - $\delta 2,4$-dienoyl-CoA isomerase & ECH1 & Q13011 & 2.0 & - \\
\hline Actin, cytoplasmic 1 & ACTB & P60709 & 1.2 & -2.0 \\
\hline ATP synthase $\alpha$ chain & ATP5A1 & P25705 & 1.2 & 2.0 \\
\hline Far upstream element binding protein 2 & KHSRP & Q92945 & 1.2 & 2.0 \\
\hline Fibroblast growth factor-5 & FGF5 & P12034 & 1.2 & 2.0 \\
\hline Heat shock cognate $71 \mathrm{kDa}$ protein & HSPA8 & P11142 & 1.2 & 2.0 \\
\hline Ran-specific GTPase-activating protein & RANBP1 & P43487 & 1.2 & 2.0 \\
\hline Creatine kinase, ubiquitous & CKMT1A & P12532 & -2.0 & -1.2 \\
\hline Enabled protein homolog & ENAH & Q8N8S7 & -2.0 & -2.0 \\
\hline Eukaryotic initiation factor $5 \mathrm{~A}$ & EIF5A & P63241 & -2.0 & -1.5 \\
\hline Far upstream element binding protein 2 & KHSRP & Q92945 & -2.0 & -2.0 \\
\hline Heterogeneous nuclear ribonucleoprotein $\mathrm{H}$ & HNRPH1 & P31943 & -2.0 & -1.5 \\
\hline Mitochondrial processing peptidase $\beta$ subunit & PMPCB & O75439 & -2.0 & -1.2 \\
\hline Translationally controlled tumor protein & TCTP & P13693 & -2.0 & - \\
\hline Chromatin assembly factor 1 subunit $\mathrm{C}$ & RBBP4 & Q09028 & -1.5 & -2.0 \\
\hline Serine-threonine kinase receptor-associated protein & STRAP & Q9Y3F4 & -1.2 & -2.0 \\
\hline Gastric-associated differentially-expressed protein YA61P & YA61 & Q9NZ23 & - & 2.0 \\
\hline Keratin, type II cytoskeletal 8 & KRT8 & P05787 & - & -2.0 \\
\hline
\end{tabular}

Listed proteins are regualted by both sms and smsdx in the same trend except actin, cytoplasmic 1 in an opposite manner.

Ctrl sms $\quad$ smsdx
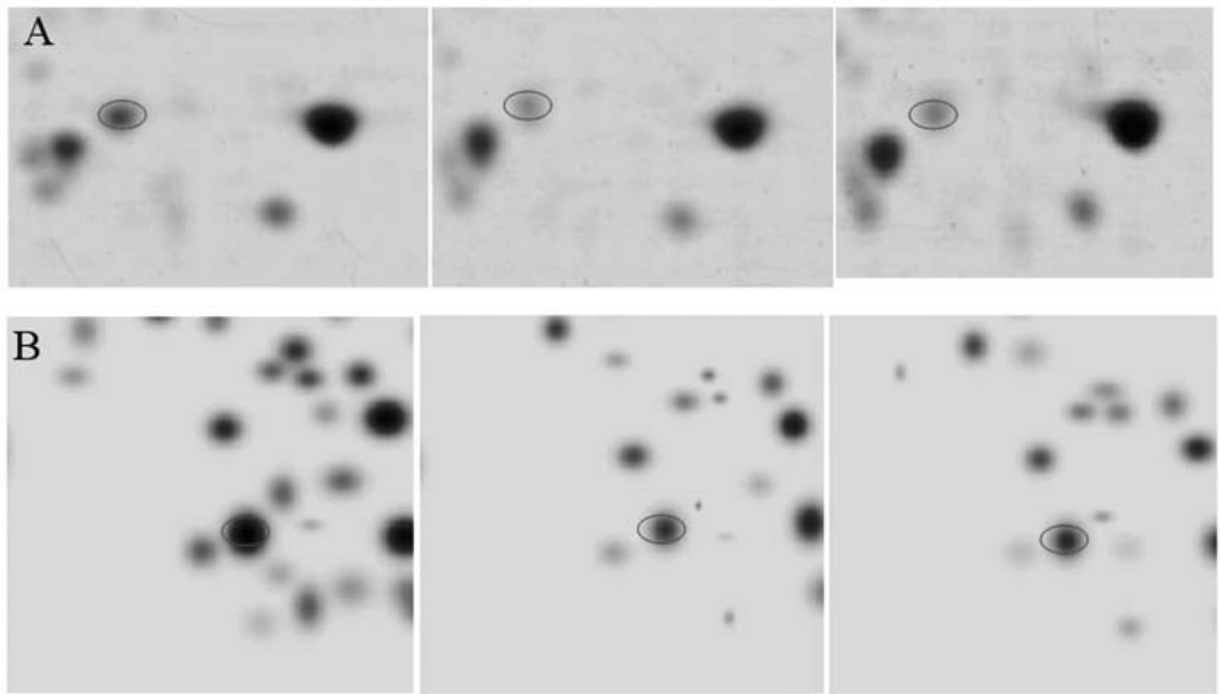

Figure 1. The identified protein expression of PRDX2 (A) and TCTP (B) (2-fold down-regulation) after treated with $1 \mathrm{nM}$ sms/smsdx for three days in the prostate cancer cell line LNCaP.

(HRPC) making anti-androgen therapy ineffective remains a significant clinical problem. Chemotherapy has only limited efficacy in AIPC and HRPC (12). During androgen-dependent progression, prostate cancer cells depend on the androgen receptor as the primary mediator of growth and survival. Certain growth factors, such as insulin-like growth-factor-1 


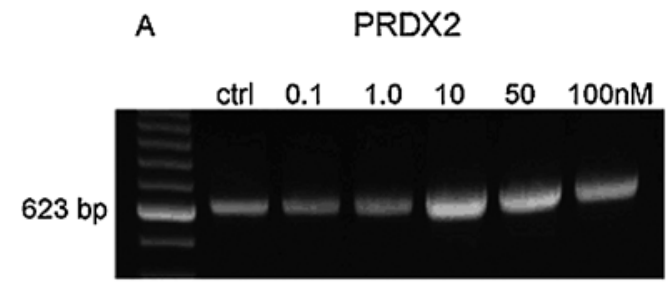

B TCTP
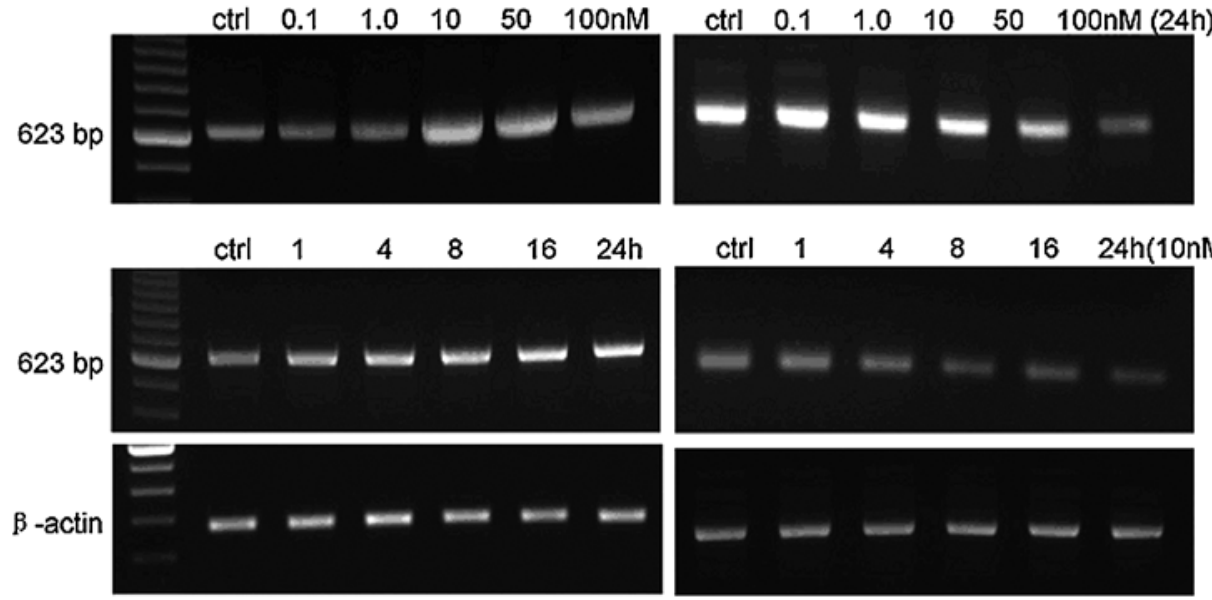

$278 \mathrm{bp}$

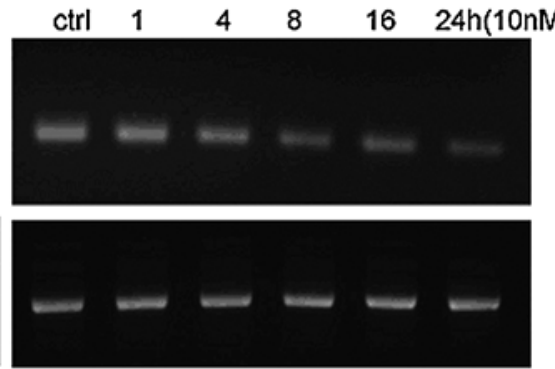

$278 \mathrm{bp}$

Figure 2. PRDX2 and TCTP mRNA expression in LNCaP cell line after incubation with smsdx. Up-regulation of PRDX2 (A) and down-regulation of TCTP (B) mRNA in smsdx treated LNCaP cell line with different concentrations $(0,0.1,1.0,10,50,100 \mathrm{nM}$ for $24 \mathrm{~h})$ (upper lane) and incubation time $(0,1,4,8$, $16,24 \mathrm{~h}$ for $10 \mathrm{nM}$ ) (middle lane); control, lower lane.

(IGF-1), can activate the androgen receptor (AR) and can therefore induce AR target genes in the absence of androgen. IGF-1 can induce a 5-fold rise in PSA secretion in LNCaP cells (13). The use of sms analogues as monotherapy in patients with HRPC has produced only modest clinical responses. However, Koutsilieris et al recently developed a therapeutic 'combination concept' i.e. combining sms with other drugs, aiming at suppressing the bioavailability of IGF-1 and downstream biological effectors. A potential mechanism for the efficacy of this combination regimen involves the abrogation of the protective effect of IGF-I on prostate cancer cells $(14,15)$. Activation of phosphotyrosine phosphatases (PTPs) by somatostatin receptors (SSTRs) represents one of the main intracellular mechanisms involved in the antiproliferative effect of sms analogues (16). The mitogen activated protein (MAP) kinase cascade represents one of the major regulators of cell growth by hormones and growth factors. Effects of sms on the MAP kinase cascade are regulated through SSTR1 (17). Kosari et al found that SSTR1 is associated with systemic progression in prostate cancer after comparisons of gene expression profiles obtained from the epithelial cells of non-neoplastic tissue, primary tumor, and metastatic tumor samples, SSTR1 was found to be the most prominent candidate prognostic biomarker in aggressive prostate cancer (18). The universal affinity of smsdx may be of importance when treating HRPC that predominantly expresses this SSTR1 subtype.

Marked quantitative differences were observed in the protein expression profiles in $\mathrm{sms} / \mathrm{smsdx}$ treated $\mathrm{LNCaP}$ and DU145 cells compared to the control cells. One third of the detected proteins were differentially expressed (PRDXs, hnRNPs, HSPs, TCTP, RKIP). Concordance in protein expression patterns were observed between smsdx and sms treated cells with strong agreement between the up- and down-regulation of proteins indicating preservation of sms effects of smsdx. Some proteins differentially expressed in androgen-dependent and androgen-independent cells can be regulated by the treatment of sms and smsdx. These proteins possibly elucidate some of the molecular mechanism of prostate cancer progression from androgen responsive to androgen refractory. The results support androgen regulatory activity on the LNCaP and DU145 cells at the level of protein.

In peroxiredoxin (PRDX) family, PRDX1, 2, 3 and 6 were identified in prostate cancer cell lines LNCaP and DU145. PRDX2 and PRDX6 were down-regulated by sms/ smsdx in LNCaP and DU145 cells. PRDX3 was up-regulated by sms/smsdx in these two cell lines. PRDX2 is known to protect cells from oxidative damage and to confer resistance to oxidative damage to cancer cells. PRDX2 had higher expression in highly metastatic prostate cancer cells (19). However, the role of PRDX2 in tumor progression is poorly understood. In this study, PRDX2 protein has a higher expression in DU145 cells compared with LNCaP cells. PRDX2 mRNA was significantly up-regulated by sms and smsdx treatment in LNCaP cells. PRDX2 protein expression was down-regulated by sms (4). The different manners of regulation by sms/smsdx in PRDXs are thought to be related to phosphorylation, overoxidation and proteolysis of PRDXs. The roles of PRDXs in regulating levels of hydrogen peroxide, an intracellular signaling molecule common to many cytokine-induced signal-transduction pathways, possibly lead to different expressions in LNCaP and DU145 cells.

The heterogeneous nuclear ribonucleoproteins (hnRNPs) have central roles in DNA repair, telomere biogenesis, cell signalling and in regulating gene expression at both transcriptional and translational levels. Yang et al found that stable transfection of hnRNPA1 in the LNCaP cells suppressed ARmediated cell growth and the expression of prostate-specific antigen, and this suppressive effect was abolished by the addition of ARA54-small interfering RNA (20). In the current study, hnRNPK, hnRNPH1, hnRNPD, hnRNPC, hnRNPA2B1 and hnRNPL were identified. hnRNPH1, hnRNPA2B1 have higher expression and hnRNPD, hnRNPC lower expression up to 2-fold change in DU145 cells compared to LNCaP cells. hnRNPH was down-regulated by sms/smsdx in DU145. hnRNPD was unregulated by sms/smsdx in DU145 and 
LNCaP cells. hnRNPA2B1 was up regulated by sms in LNCaP but not in DU145 cells. The hNRNPs were regulated by $\mathrm{sms} / \mathrm{smsdx}$ in different manner, sometime up sometime down. Their differential expression of hnRNPs sheds light on the underlying transition mechanism from prostatic androgendependence to androgen-independence and the different aggressive and metastatic abilities between $\mathrm{LNCaP}$ and DU145 cells.

Heat shock proteins (HSPs) have strong cytoprotective effects and behave as molecular chaperones for other cellular proteins. Several of these proteins have been demonstrated to directly interact with components of the cell signalling pathways, for example, those of the tightly regulated caspasedependent programmed cell death machinery, upstream, downstream and at the mitochondrial level (21). Among the different HSPs, HSP27 and HSP70 are the most strongly induced after stresses such as anticancer drugs, oxidative stress or irradiation. Both HSP27 and HSP70 are abundantly expressed in cancer cells and therefore have been suggested as important prognostic factors in malignant diseases (22). In the current study, some of HSPs were regulated by sms/ smsdx. For example, HSP60/HSPD1 was up-regulated by both sms and smsdx in DU145. Since it is known that HSPs bind to and inactivate ARs, one could assume that increased concentration of HSP60 may block the binding of androgens to the receptor.

The translationally controlled tumor protein (TCTP) is an abundantly expressed protein. Arcuri et al found expression of TCTP in the human prostate and in prostate cancer cells (23), and suggested the involvement of the protein in keyprocesses such as apoptosis, cellular differentiation, and in the control of sperm functions. TCTP was found to antagonize apoptosis by inserting into the mitochondrial membrane and inhibiting Bax dimerization (24). So deregulation of TCTP by smsdx in the current study suggested its possible involvement in the sms analogue cytostatic effect via mitochondrialmediated pathway.

Raf kinase inhibitory protein (RKIP) was found to regulate activation of MAPK, NF- $\mathrm{BB}$ and $\mathrm{G}$ protein coupled receptors (GPCRs) (25). RKIP plays an important role in cancer invasion and metastasis (26). Its levels were found to be reduced or absent in variants of established cell lines derived from metastatic prostate cancer (27). Overexpression of RKIP sensitizes tumor cells to chemotherapeutic drug-induced apoptosis (28). Fougner et al found a significant correlation between the adenoma RKIP level and the clinical effect of Octreotide in addition to the SSTR2a protein expression (29). Therefore, a synergistic inhibitory effect on the MEK-ERK pathway is a possible mechanism for the association between adenoma RKIP level and the clinical efficacy of sms analogues. In the present study, RKIP was found in a higher level of expression in androgen-dependent $\mathrm{LNCaP}$ cells, but lower expression in androgen-independent DU145 cells. Low RKIP levels in DU145 were suggested to be correlated with enhanced extracellular signal-regulated-kinase (ERK)/MAPK pathway activation. This suggested RKIP has a possible role in the transition of androgen dependency and is a potential target for prostate cancer.

In conclusion, the proteomic analysis has yielded quantitative and qualitative results elucidating the effect of androgen deprivation on the proteome of prostatic cancer $\mathrm{AD}$ and $\mathrm{AI}$ cell lines. From the present results it seems reasonable to assume that sms/smsdx affects the mitochondria of $\mathrm{LNCaP}$ and DU145 cells in a way that eventually triggers mitochondrialmediated apoptosis. Induced expression of SSTRs and regulation of certain proteins by $\mathrm{sms} / \mathrm{smsdx}$ in the present study suggest that sms/smsdx exerts its effects on prostate cancer cells via the MAPK pathway and by regulating the activities of PTPs.

\section{Acknowledgements}

Supported by The Cancer Society in Stockholm, The King Gustav V Jubilee Fund, Stockholm, The Swedish Cancer Society and Mr. Svante Wadman, Stockholm. The National Natural Science Foundation of China (30772294/C1612).

\section{References}

1. Parker SL, Tong T, Bolden S and Wingo PA: Cancer statistics 1996. CA Cancer J Clin 46: 5-27, 1996.

2. Denis L and Murphy GP: Overview of phase III trials on combined androgen treatment in patients with metastatic prostate cancer. Cancer 72 (Suppl. 12): S3888-S3895, 1993.

3. Liu Z, Márquez M, Nilsson S, Holmberg AR and Alaiya AA: Proteomic analysis of a human prostate cancer cell line after incubation with a novel somatostatin 14 derivative. Cancer Genomics Proteomics 2: 347-352, 2005.

4. Liu Z, Bengtsson S, Krogh M, et al: Somatostatin effects on the proteome of the LNCaP cell-line. Int J Oncol 30: 1173-1179, 2007.

5. Liu Z, Márquez M, Nilsson S and Holmberg AR: Incubation with somatostatin, 5-aza decitabine and trichostatin up-regulates somatostatin receptor expression in prostate cancer cells. Oncol Rep 20: 151-154, 2008.

6. Brevini TA, Bianchi R and Motta M: Direct inhibitory effect of somatostatin on the growth of the human prostatic cancer cell line LNCaP: possible mechanism of action. J Clin Endocrinol Metab 77: 626-631, 1993.

7. Franzén B, Linder S, Okuzawa K, Kato H and Auer G: Nonenzymatic extraction of cells from clinical tumor material for analysis of gene expression by two-dimensional polyacrylamide gel electrophoresis. Electrophoresis 14: 1045-1053, 1993.

8. Rabilloud T, Vuillard L, Gilly C and Lawrence JJ: Silver-staining of proteins in polyacrylamide gels: a general overview. Cell Mol Biol 40: 57-75, 1994.

9. Garrels JI: The QUEST system for quantitative analysis of twodimensional gels. J Biol Chem 264: 5269-5282, 1989.

10. Damber JE and Aus G: Prostate cancer. Lancet 371: 1710-1721, 2008.

11. Ferlay J, Autier P, Boniol M, Heanue M, Colombet M and Boyle P: Estimates of the cancer incidence and mortality in Europe in 2006. Ann Oncol 18: 581-592, 2007.

12. Machiels JP, Mazzeo F, Clausse M, et al: Prospective randomized study comparing docetaxel, estramustine, and prednisone with docetaxel and prednisone in metastatic hormone-refractory prostate cancer. J Clin Oncol 26: 5261-5268, 2008.

13. Culig Z, Hobisch A, Cronauer MV, et al: Androgen receptor activation in prostatic tumor cell lines by insulin-like growth factor-I, keratinocyte growth factor, and epidermal growth factor. Cancer Res 54: 5474-5478, 1994.

14. Koutsilieris M, Mitsiades C, Dimopoulos T, Ioannidis A, Ntounis A and Lambou T: A combination therapy of dexamethasone and somatostatin analog reintroduces objective clinical responses to LHRH analog in androgen ablationrefractory prostate cancer patients. J Clin Endocrinol Metab 86: 5729-5736, 2001.

15. Koutsilieris M, Mitsiades CS, Bogdanos J, et al: Combination of somatostatin analog, dexamethasone, and standard androgen ablation therapy in stage D3 prostate cancer patients with bone metastases. Clin Cancer Res 10: 4398-4405, 2004.

16. Florio T: Somatostatin/somatostatin receptor signalling, phosphotyrosine phosphatases. Mol Cell Endocrinol 286: 40-48, 2008. 
17. Florio T, Thellung S, Arena S, et al: Somatostatin receptor 1 (SSTR1)-mediated inhibition of cell proliferation correlates with the activation of the MAP kinase cascade: role of the phosphotyrosine phosphatase SHP-2. J Physiol 94: 239-250, 2000 .

18. Kosari F, Munz JM, Savci-Heijink CD, et al: Identification of prognostic biomarkers for prostate cancer. Clin Cancer Res 14: 1734-1743, 2008.

19. Wu M, Bai X, Xu G, et al: Proteome analysis of human androgen-independent prostate cancer cell lines: variable metastatic potentials correlated with vimentin expression. Proteomics 7: 1973-1983, 2007.

20. Yang Z, Chang YJ, Miyamoto H, et al: Suppression of androgen receptor transactivation and prostate cancer cell growth by heterogeneous nuclear ribonucleoprotein A1 via interaction with androgen receptor coregulator ARA54. Endocrinology 148: 1340-1349, 2007.

21. Lanneau D, Brunet M, Frisan E, Solary E, Fontenay M and Garrido C: Heat shock proteins: essential proteins for apoptosis regulation. J Cell Mol Med 12: 743-761, 2008.

22. Garrido C, Brunet M, Didelot C, Zermati Y, Schmitt E and Kroemer G: Heat shock proteins 27 and 70: antiapoptotic proteins with tumorigenic properties. Cell Cycle 5: 2592-2601, 2006.
23. Arcuri F, Papa S, Carducci A, et al: Translationally controlled tumor protein (TCTP) in the human prostate and prostate cancer cells: expression, distribution, and calcium binding activity. Prostate 60: 130-140, 2004.

24. Susini L, Besse S, Duflaut D, et al: TCTP protects from apoptotic cell death by antagonizing bax function. Cell Death Differ 15: 1211-1220, 2008.

25. Zeng L, Imamoto A and Rosner MR: Raf kinase inhibitory protein (RKIP): a physiological regulator and future therapeutic target. Expert Opin Ther Targets 12: 1275-1287, 2008.

26. Keller ET, Fu Z, Yeung K and Brennan M: Raf kinase inhibitor protein: a prostate cancer metastasis suppressor gene. Cancer Lett 207: 131-137, 2004.

27. Fu Z, Smith PC, Zhang L, et al: Effects of raf kinase inhibitor protein expression on suppression of prostate cancer metastasis. J Natl Cancer Inst 95: 878-879, 2003.

28. Chatterjee D, Bai Y, Wang Z, et al: RKIP sensitizes prostate and breast cancer cells to drug-induced apoptosis. J Biol Chem 279: 17515-17523, 2004.

29. Fougner SL, Bollerslev J, Latif F, et al: Low levels of raf kinase inhibitory protein in growth hormone-secreting pituitary adenomas correlate with poor response to octreotide treatment. J Clin Endocrinol Metab 93: 1211-1216, 2008. 